\title{
WIE INTEGRIERT MAN ROM IN DIE POLIS? DER KULT DES SENATS IN KLEINASIEN*
}

\author{
Günther Schörner
}

\section{Integration: Akteure und Handlungen}

Möchte man Integration im Imperium Romanum untersuchen, so ist nicht nur zu analysieren, auf welche Weise die Provinzen und ihre Bewohner in das Römische Reich integriert wurden, sondern auch, wie Rom selbst in das provinziale Umfeld integriert wurde. ${ }^{1}$ In diesem Fall wird nicht nach reichsweiter Integration, sondern nach lokal begrenzter, je nach politischer Organisation oft nach Integration auf städtischer Ebene gefragt. Dabei sollte man sich nicht darauf beschränken festzustellen, was integriert wurde, da die Frage nach dem Wie mindestens genau so wichtig ist, das heißt welche Aktionen erfolgten und wer diese veranlasste. Im Folgenden sollen Akteure und Handlungen einer solchen Integration an einem konkreten Beispiel aufgezeigt werden, nämlich der Integration der spezifisch römischen Institution 'Senat' in den kultischen Kontext kleinasiatischer Poleis. Es stehen dabei weniger Fragen der Kultausübung im Mittelpunkt, sondern vielmehr die Prozeduren und Aktionen sowohl auf römischer als auch auf kleinasiatischer Seite, die unternommen werden, um für eine dezidiert römische Institution wie den Senat Platz innerhalb der Poleis zu schaffen, d. h. ihn in dieses spezifisch sozio-kulturelle Umfeld einzubinden.

* Ich danke den Organisatoren des Workshops Impact of Empire, vor allem S. Benoist, S. Demougin, und O. Hekster für die Einladung nach Lille. Wichtige Thesen dieses Vortrags wurden bereits in Amsterdam, Erfurt und Würzburg vorgetragen. Für Diskussionsbeiträge danke ich A. Chankowski (Lille), L. de Blois (Nijmegen), S. Demougin (Paris), J. Fabricius (Berlin), M. Meyer (Wien), F. Naerebout (Leiden), J. Rüpke (Erfurt) und Th. Schäfer (Tübingen).

1 Zum Begriff der Integration: G. Alföldy, 'Die Romanisation - Grundbegriff oder Fehlbegriff? Überlegungen zum gegenwärtigen Stand der Erforschung von Integrationsprozessen im römischen Weltreich', in Z. Visy (ed.), Limes XIX. Proceedings of the XIXth International Congress of Roman Frontier Studies (Pécs 2005), 25-56; R. Roth, 'Roman culture between homogeneity and integration', in R. Roth, J. Keller (eds.), Roman by integration: Dimensions of group identity in material culture and text (Journal of Roman archaeology Supplementary Series 66) (Portsmouth, RI 2007), 7-10; zusammenfassend zur Diskussion: D. Mattingly, Imperialism, power, and identity: experiencing the Roman Empire (Princeton, NJ 2010) 3-42; vgl. in diesem Band den Beitrag von F. Naerebout. 
Im Unterschied zu den anderen römischen Institutionen besitzt der Senat als Objekt kultischer Verehrung Eigenheiten, die eine deutlichere Differenzierung zwischen römischer und griechischer Seite zulassen und somit die Funktionsweise der Integration leichter nachvollziehbar machen. ${ }^{2}$ Das wichtigste Charakteristikum ist sprachlicher Natur: Im Unterschied zum lateinischen senatus ist das griechische $\Sigma u ́ \gamma x \lambda \eta \tau o s$ feminin, so dass die

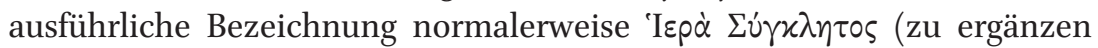
ist: Bov $\lambda$ ) lautet. Der Wechsel des Genus hatte natürlich Konsequenzen für das Erscheinungsbild, das die Senatsgottheit in Kleinasien annahm, da aufgrund des grammatikalischen Geschlechts in Kleinasien an und für sich eine weibliche Personifikation $\Sigma$ YNK $\Lambda H T O \Sigma$ BOY $\Lambda$ H bzw. IEPA $\Sigma$ YNK $\Lambda$ HTO $\Sigma$ zu erwarten ist. ${ }^{3}$ Folglich ist es verständlich, dass trotz der hauptstädtischen Herkunft der Institution nicht die stadtrömische Verkörperung als Genius Senatus übernommen wurde. Der Genius Senatus besitzt spätestens seit flavischer Zeit eine feste Ikonographie:4 Er wird stets als älterer bärtiger Mann in Toga mit idealen Gesichtszügen und langen Locken dargestellt. ${ }^{5}$ Als Personifikationen der res publica tritt der Genius Senatus regelhaft zusammen mit dem deutlich jugendlicher wiedergegebenen Genius Populi Romani auf, ${ }^{6}$ eines der frühesten Zeugnisse sind die so genannten Cancelleria-Reliefs. ${ }^{7}$ Grundsätzlich unterscheidet

2 Zum Kult des Senats/Synkletos in Kleinasien grundlegend: G. Forni, IEPA e $\Theta E O C$ CVNKLETOC. Un capitolo dimenticato nella storia del Senato Romano (Atti della Accademia Nazionale die Lincei, Memorie classe di scienze morali, storiche e filologiche ser. VIII, vol. V.3) (Rom 1953); G. Forni, 'Il culto del Senato di Roma', in Epigrafia e ordine senatorio. Atti del colloquio AIEGL (Roma, 14-20 maggio 1981) (Rom 1982), 14-18; D. Kienast, 'Der heilige Senat: Senatskult und „kaiserlicher" Senat', Chiron 15 (1985), 253-282.

3 Kienast 1985, a.a.O. (Anm.2), 266 f. Vgl. auch A. Chaniotis, 'The dynamics of rituals in the Roman Empire', in O. Hekster, S. Schmidt-Hofner, C. Witschel (eds.), Ritual Dynamics and Religious Change in the Roman Empire. (Proceedings of the Eighth Workshop of the International Network Impact of Empire, Heidelberg, July 5-7, 2007) (Leiden 2009), 4.

4 H. Kunckel, Der römische Genius. 20. Ergänzungsheft der Römischen Mitteilungen (Heidelberg 1974), 37-42.

5 Vgl. auch die Beschreibung des personifizierten Senats, der Traian im Traum erscheint: Dio Cass., 68.5.1.

6 Kunckel 1974, a.a.O. (Anm.4), 33-37, 40-43; J.R. Fears, 'O $\Delta$ HMO $\Sigma$ O P $\Omega$ MAI $\Omega N$ Genius Populi Romani', Mnemosyne 31 (1978), 275-279.

7 Zu den Reliefs allgemein G. Koeppel, 'Die historischen Reliefs der römischen Kaiserzeit II: Städtrömische Denkmäler unbekannter Bauzugehörigkeit aus flavischer Zeit', Bonner Jahrbücher 184 (1984), 5-9, 28-34; N. Hannestad, Roman Art and Imperial Policy (Aarhus 1988), 132-139; H. Herzog, 'Die Cancelleriareliefs', Bollettino. Monumenti musei a gallerie pontificie 21 (2001), 103-147; speziell zu den beiden Genien: J. Béranger, 'Les 
sich der Genius Senatus durch Kleidung und Barttracht signifikant von den meisten anderen Genien, die als junger Mann wiedergegeben sind. ${ }^{8}$

Diese geläufige stadtrömische Erscheinungsform wurde - wie zu erwarten - in Kleinasien nicht verwendet, jedoch wurde auch kein dem griechischen Sprachgebrauch entsprechendes weibliches Bild konzipiert in Analogie zu Verkörperungen von Ratsversammlungen wie Boulê ${ }^{9}$ oder Gerousia. ${ }^{10}$ Geschaffen wurde eine neuartige Personifikation, ${ }^{11}$ anhand deren Ikonographie und Verbreitung gezeigt werden kann, mit welchen Schritten eine römische Institution in das Leben der Provinz Asia integriert wird. ${ }^{12}$

Génies du sénat et du peuple romain et les reliefs flaviens de la Cancelleria', in M. Renard, R. Schilling (eds.), Hommages à Jean Bayet (Brüssel 1964), 76-88.

8 z.B. auch die vielen privaten Genien: Kunckel 1974, a.a.O. (Anm.4) 42 f. (mit Beispielen); allgemein Lexicon Iconographicum Mythologiae Classicae VIII (München-Zürich 1997), 599-607 s.v. Genius (I. Romeo); vgl. auch die Ikonographie der Lokalgenien z.B. L. da Silva Fernandes, 'Genii, Lares e Tutela na provincia da Lusitânia', in J. Cardim Ribeiro (ed.), Religiôes da Lusitânia: loquuntur saxa (Lissabon 2002), 179-188.

9 Zur Ikonographie der Boulê allgemein Lexicon Iconographicum Mythologiae Classicae III (München, Zürich 1984), 145-147 s.v. Boulê (V. Komminos) (mit weiterer Lit.); zur Personifikation in klassischer Zeit: W. Messerschmidt, PROSOPOPOIIA. Personifikationen politischen Charakters in spätklassischer und hellenistischer Kunst (Köln, Weimar, Wien 2003), 48-50; zur Personifikation auf kleinasiatischen Münzen der römischen Kaiserzeit: F. Missere, 'Iconografia della Boulê e del Demos nella monetazione imperiale greca dell'Asia minore', Rivista Italiana di Numismatica 92 (1990), 76-87; Statuen epigraphisch belegt: Th. Pekáry, 'Statuen in kleinasiatischen Inschriften', in S. Sahin, E. Schwertheim, J. Wagner (eds.), Studien zur Religion und Kultur Kleinasiens. Festschrift für Friedrich Karl Dörner zum 65. Geburtstag am 28. Februar 1976 (Leiden 1978), 739.

10 Lexicon Iconographicum Mythologiae Classicae VIII (München, Zürich 1997), 608 s.v. Gerousia (S. Schultz); zur Institution: J.H. Oliver, The sacred Gerusia (6. Supplement Hesperia) (Princeton 1941).

11 Für die Definition einer Personifikation wird gefolgt: A.C. Smith, Polis and personification in Classical Athenian art (Leiden, Boston 2011), 2: „... personification as representation of a thing, place, or abstraction as a person, where person may be human, mythical, and/or divine." $\mathrm{Zu}$ eng und im unseren Kontext nicht anzuwenden ist die Definition von Personifikation durch B. Borg, Der Logos des Mythos. Allegorien und Personifikationen in der frühen griechischen Kunst (München 2002), 49 f.: Personifikationen sind „.... alle anthropomorphen und anthropopsychen Gestalten..., deren Namen gleichzeitig als abstraktes Substantiv oder Bezeichnung für einen unbelebten Gegenstand existiert". Beibehalten wird jedoch ihre Unterscheidung von 'Personifizierung' als der Aktion und 'Personifikation' als dem Produkt: Borg a.a.O., $5^{2}$.

12 Zur Personifikation von Synkletos neben der in Anm.2 genannten Lit. speziell: Lexikon Iconographicum Mythologiae Classicae VII (München, Zürich 1994), 828 f. s.v. Synkletos (F. Canciani). 


\section{Kult und Kultbild: Römischer Entwurf - kleinasiatische Übernahme}

Die kultische Verehrung eines politischen Gremiums erscheint zunächst noch erstaunlicher als die einer einzelnen Person wie eines Kaisers, ${ }^{13}$ doch ist der römische Senat als Kultempfänger zweifelsfrei nachgewiesen. ${ }^{14} \mathrm{Zu}$ berücksichtigen ist dabei, dass vor allem die Handlungsfähigkeit und die Einflussmöglichkeiten entscheidend für die Etablierung kultischer Verehrung waren. ${ }^{15}$ Diese waren im Falle des römischen Senats für die kleinasiatischen Städte aus deren Perspektive sicherlich gegeben: In Asia als einer senatorischen Provinz wurde der Senat als wichtige politische und legislatorische Instanz wahrgenommen, insbesondere erfolgte die Verleihung von Ehrentitel wie Neokoros, um die sich ein wichtiger Aspekt des städtischen Lebens in Kleinasien drehte, durch den Senat. ${ }^{16}$ Es ist also nur verständlich, wenn der Senat noch in der Kaiserzeit sowohl im Verkehr mit Rom als auch unter den Poleis als entscheidende Größe empfunden wurde. ${ }^{17}$

Obwohl der Senat auf städtischer Ebene sicher bereits unter Augustus verehrt wurde, erfolgte die Einrichtung eines provinzweiten Kultes unter Tiberius schließlich in Smyrna, wo für Tiberius, die Kaisermutter Livia

13 Zum Kaiserkult: S.R.F. Price, Rituals and Power: The Roman Imperial Cult in Asia Minor (Cambridge 1984); M. Clauss, Kaiser und Gott: Herrscherkult im römischen Reich (Stuttgart 1999); C. Ando, Imperial ideology and provincial loyalty in the Roman empire (Berkeley 2000); I. Gradel, Emperor worship and Roman religion (Oxford 2002); T. Witulski, Kaiserkult in Kleinasien. Die Entwicklung der kultisch-religiösen Kaiserverehrung in der römischen Provinz Asia von Augustus bis Antoninus Pius (Göttingen 2011²); (jeweils mit weiterer Lit.).

14 Zeugnisse gesammelt bei Forni 1953, a.a.O. (Anm.2), 67-79; Forni 1982, a.a.O. (Anm.2), 14-18; Kienast 1985 a.a.O. (Anm.2) 268 Anm.66, 68.

15 Immer noch grundlegend: E. Bickerman, 'Consecratio', in E. Bickerman, C. Habicht (eds.), Le culte des souverains dans l'empire romain (Entretiens sur l'Antiquité classique 19) (Genf 1972), 1-26; vgl. auch G. Woolf, 'Divinity and power in ancient Rome', in N. Brisch (ed.), Religion and power. Divine kingship in the ancient world and beyond (Chicago 2008), $235^{-252 .}$

16 Zur Neokorie zusammenfassend: B. Burrell, Neokoroi. Greek Cities and Roman Emperors (Leiden, Boston 2004); Senat in dieser Rolle gewürdigt und auf Münze festgehalten: K. Harl, Civic coins and civic politics in the Roman east A.D. 180-275 (Berkeley, London 1987), 61 Taf. 27,6. Grundlegend zur Wahrnehmung des Senats aus Sicht einer kleinasiatischen Polis am Beispiel Ephesos: G.M. Rogers, The sacred identity of Ephesos. Foundation myths of a Roman city (London, New York 1991), $91 \mathrm{f}$.

17 Zum Senat in der Kaiserzeit: R.J.A. Talbert, The senate of Imperial Rome (Princeton, NJ 1984); zur Rolle des Senats in Kleinasien: Harl 1987, a.a.O. (Anm.16), 71-75. 
und den Senat ein Tempel errichtet wurde. ${ }^{18}$ Über die Umstände dieser Kultgründung berichtet vor allem Tacitus: ${ }^{19}$

Dieser Entscheidung halber und, weil auch im vorigen Jahre C. Silanus zur Strafe gezogen worden war, beschlossen die kleinasiatischen Städte, zu Ehren des Tiberius, seiner Mutter und des Senats einen Tempel zu erbauen. Der Bau wurde auch gestattet, und der junge Nero war es, der den Senatoren und seinem Großvater den Dank dafür aussprach - unter freudiger Bewegung der Zuhörer, die Germanicus noch lebhaft im Gedächtnis hatten und jetzt ihn selber zu sehen und hören meinten.

Kienast konnte in überzeugender Interpretation dieser Passage darauf hinweisen, dass der Kult von Synkletos in diesem Kontext auf Tiberius selbst zurückgeht, ${ }^{20}$ dessen Hochachtung des Senats auch durch einen von Sueton überlieferten Ausspruch belegt wird. ${ }^{21}$ Die Kommunikation und Interaktion zwischen Kaiser und Gesandtschaften, das heißt zwischen verschiedenen Erwartungshorizonten ist bekanntermaßen sehr kompliziert, doch ist davon auszugehen, dass man die Spezifikation, d.h. die Bestandteile dieses Kaiserkultkomplexes auf Rom zurückführen kann. Die römische Einwirkung ging jedoch noch weiter: ${ }^{22}$

Bei der Abstimmung des Senats erhielt denn auch Smyrna den Vorzug, und Vibius Marsus beantragte, man möge dem M. Lepidus, dem die Provinz Asien zugefallen war, einen überzähligen Legaten mitgeben der die Aufsicht über den Tempelbau zu führen habe. Lepidus lehnte aus Bescheidenheit ab, diesen Legaten selber zu ernennen; das Los fiel auf den ehemaligen Prätor Valerius Naso.

Die deutliche römische Einflussnahme bei der Konzeption dieser Kultstätte ist $\mathrm{m}$. E. nun auch ikonographisch zu belegen: Die Errichtung dieses provinzialen Kaiserkulttempels wird auch durch eine Münze aus Smyrna memoriert (Abb. 1), die auf der Rückseite einen tetrastylen Tempel zeigt, in dem ein Togatus capite velato, also Tiberius selbst als der erstgenannte Kultempfänger, mit einer Schöpfkelle, einem simpuvium, in der rechten Hand steht. ${ }^{23}$ Auf der Vorderseite sind zwei einander zugewandte Büsten

18 Zur voraugusteischen Verehrung des Senats auf städtischer Ebene: Chr. Habicht, 'Samos weiht eine Statue des Populus Romanus', Athenische Mitteilungen 105 (1990), 262 mit Anm.24. (mit Beleg).

19 Tac., Ann. 4.15; 55 f. (Übersetzung: W. Sontheimer).

20 Kienast 1985, a.a.O. (Anm.2), $261 \mathrm{f}$.

21 Suet., Tib. 29.

22 Tac., Ann. 4.56.3 (Übersetzung: W. Sontheimer): Kienast 1985, a.a.O. (Anm.2), 260.

23 Zur Münze: D. Klose, Die Münzprägung von Smyrna in der römischen Kaiserzeit (Berlin 1987), 212-214 nº XXVI 1-35 Taf. 18. 19; A. Burnett, M. Amandry, P.P. Ripollès, Roman 

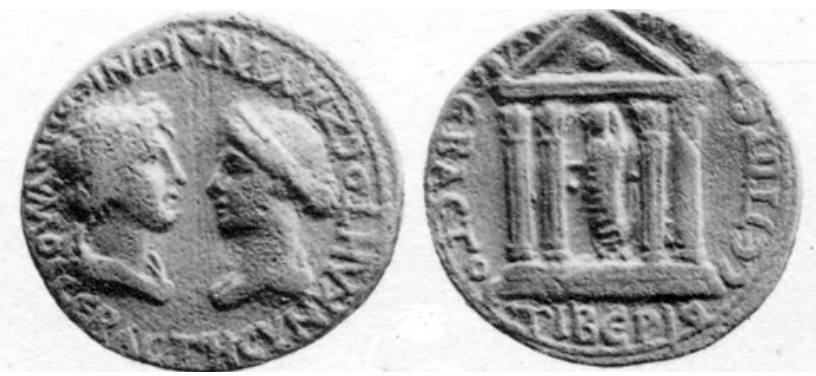

Abb. 1: AE Smyrna: Synkletos und Livia; Tiberius in Tempel

dargestellt, die einer Frau mit Diadem und Nackenknoten und die eines jungen Mannes mit langem Haar. Laut Beischrift handelt es sich um die Darstellungen der Sebaste Livia und der Senatsgottheit Synkletos als die beiden anderen Tempelinhaber, wobei dieser eindeutig nicht weiblich, sondern männlich dargestellt wurde, freilich auch nicht als alter Mann wie der - später bezeugte - römische Genius Senatus. ${ }^{24}$

Die ikonographische Konzeption des Togatus, das heißt der TiberiusStatue, so wie sie auf der Münze wiedergegeben ist, erweist sich eindeutig als stadtrömisch. Dafür sprechen nicht nur die Toga, sondern vor allem die Verhüllung des Hinterkopfes, die velatio capitis, als typisches Zeichen eines nach dem ritus Romanus vollzogenen Kultaktes und das simpuvium. ${ }^{25}$ Dieses Sakralgerät ist charakteristisch für das collegium der pontifices und wurde insignienhaft verwendet, wie Monumente unterschiedlicher Gattungen belegen. ${ }^{26}$ Der Darstellungstyp eines Togatus capite velato mit simpuvium ist für Augustus auf einem stadtrömischen Aureus des Münzmeisters C. Marius aus dem Jahr 13 v. Chr. bezeugt;27 In Kleinasien wurde ein ähnliches Münzbild erstmals in Ilion mit der

provincial Coinage I: From the death of Caesar to the death of Vitellius (London 1992), 419 $\mathrm{n}^{\circ}$ 2469; S.J. Friesen, Twice neokoros. Ephesos, Asia, and the cult of the Flavian imperial family (Leiden 1993), 20 Taf. 3; Burrell 2004, a.a.O. (Anm.16), 39 f.

${ }^{24}$ Hier zu Anm.4.

25 Grundlegend zur velatio capitis: H. Freier, Caput velare (Tübingen 1963).

${ }^{26}$ Zwierlein-Diehl, 'Simpuvium Numae', in H.A. Cahn (ed.), Tainia. Roland Hampe zum 70. Geburtstag am 2. Dezember 1978 dargebracht von Mitarbeitern, Schülern und Freunden (Mainz 1980), 405-422; A.V. Siebert, Instrumenta sacra. Untersuchungen zu römischen Opfer-, Kult- und Priestergeräten (Berlin 1999), 132-134; 236-239.

27 Siebert 1999, a.a.O. (Anm.26), 324 Mü 35. 
Beischrift $\sum \dot{\varepsilon} \beta \alpha \sigma \tau o \zeta$ verwendet, ${ }^{28}$ also in einer Stadt mit bekannt engen Beziehungen zu Rom. ${ }^{29}$

Folgt die Darstellung des Tiberius als togatus capite velato römischwestlichen Vorstellungen, so gilt das dann mit großer Wahrscheinlichkeit ebenso für die Büsten der beiden anderen Kultempfänger auf der Rückseite. So entspricht das Porträt der Livia dem üblichen römischen Typus mit Mittelscheitel. ${ }^{30}$ Die jugendlich-männliche Senatsgottheit dürfte deshalb ebenfalls auf eine römische Konzeption zurückgehen, wie es auch dem lateinischen Sprachgebrauch entsprechend sein Geschlecht nahe legt. Es ist durchaus möglich, dass die Personifikation für die Kultstatue in Smyrna erfunden wurde. Dass es bereits einen jugendlichen Mann als Personifikation von Synkletos in augusteischer Zeit gegeben hat, ist unwahrscheinlich. ${ }^{31}$ Erstaunlicherweise entspricht die Ikonographie aber nicht dem - späteren - Genius Senatus-Typus, sondern nach römischen Begriffen viel eher dem Genius Populi Romani. ${ }^{32}$ Die besten Parallelen für die jugendliche, langhaarige Büste sind somit Darstellungen entsprechender Genii wie auf einem der berühmten Boscoreale-Becher ${ }^{33}$ oder wohl bereits der Ara Pacis. ${ }^{34}$ Die Entstehung des stadtrömischen Genius-Bildes haben H. Kunkel und H. Jucker untersucht: ${ }^{35}$ Jucker konnte zeigen, dass bereits im 1. Jh. v. Chr. gelegentlich unbärtige Genien vorkommen, aber keine durchlaufende Bildtradition nachzuweisen ist. ${ }^{36}$ Der neue, dann schnell übliche Typ des jugendlichen Genius mit schulterlangem Haar

28 Burnett, Amandry, Ripollès 1992, a.a.O. (Anm.23), 391 n $^{\circ} 2311$ (mit weiterer Lit.).

29 D. Hertel, Die Mauern von Troia. Mythos und Geschichte im antiken Ilion (München 2003), 274-284.

30 Zum Typ: P. Zanker in K. Fittschen - P. Zanker, Katalog der römischen Porträts in den Capitolinischen Museen und den anderen Kommunalen Sammlungen der Stadt Rom (Mainz 1983), 3-5 $n^{\circ}$ 3. Livia ersetzt die ansonsten für die Münzprägung Smyrnas übliche Statue der Aphrodite Stratonikis: M. Meyer, 'Die Aphrodite Stratonikis in Smyrna und die Asylie der Stadt', Jahrbuch für Numismatik und Geldgeschichte 6o (2010), 52-54.

31 Lokalprägungen aus Lampsakos in Mysien zeigen eine Kaiserbüste und die Büste

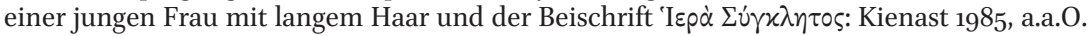
(Anm.2), 257 Taf. I 5 .

32 S.o. Anm.5.

33 A.L. Kuttner, Dynasty and empire in the age of Augustus. The case oft he Boscoreale cups (Berkeley, Los Angeles 1995), passim; zum Genius populi Romani speziell: ebenda 18-22 Abb. 1.

34 G. Koeppel, 'Die historischen Reliefs der römischen Kaiserzeit V: Ara Pacis Augustae Teil 1', Bonner Jahrbücher 187 (1987), $15 \mathrm{n}^{\circ}$ 4, 3 .

35 Kunckel 1974, a.a.O. (Anm.4), 33-37; H. Jucker, 'Ipse suos Genius adsit visurus honores!', in G. Schwarz, E. Pochmarski (eds.), Classica et provincalia. Festschrift Erna Diez (Graz 1978), 93-96; Fears 1978, a.a.O. (Anm.6), 276-279.

36 Jucker 1978, a.a.O. (Anm.35), 94. 
wurde folglich erst unter Augustus ausgebildet, während der Genius Senatus in Rom als Personifikation des Senats eine noch spätere Erfindung darstellt. ${ }^{37}$

Es spricht somit alles dafür, dass die Personifikation von Synkletos für den Tempel in Smyrna erfunden wurde. Allein aufgrund des gewählten Geschlechts ist die Konzeption eindeutig auf römische Vorstellungen zurückzuführen; auch hinsichtlich der Kommunikation ist davon auszugehen, dass senatus der terminologische Ausgangspunkt war. Es liegt deshalb nahe, Valerius Naso eine entscheidende Rolle zuzubilligen. Ob bei der Bilderfindung der jugendlichen männlichen Personifikation rein instrumentell auf griechische Ideen zurückgegriffen wurde, darf bezweifelt werden. Als Anregung kommt nur die Personifikation des Demos in Frage, weil sie in der kleinasiatischen Münzprägung der Kaiserzeit der des jugendlichen langhaarigen Synkletos sehr ähnelt. ${ }^{38}$ Die klassische Bildvorstellung - der Demos als älterer bärtiger Bürger - wird jedoch kontinuierlich bis in die hellenistische und römische Zeit beibehalten, ${ }^{39}$ so dass die Popularität des jugendlichen unbärtigen Demos ebenfalls auf römischen Einfluss in Anlehnung an den Genius Populi Romani zurückzuführen sein dürfte. ${ }^{40}$

\section{Die Personifikation von Synkletos in Kleinasien: ikonographischen Optionen}

Im Hinblick auf die Ikonographie des Synkletos ist somit davon auszugehen, dass speziell für den Export nach Kleinasien von römischer Seite ein Bild des Senates geschaffen wurde, das weder in Rom noch in Kleinasien direkte Vorbilder hatte; jedenfalls wurde die Büste bzw. Kultstatue aus aktuellen römischen Bestandteilen und unter römischer Aufsicht geschaf-

37 So auch H. Wrede, 'Der genius populi Romani und das Fünfsäulendenkmal der Tetrarchen', Bonner Jahrbücher 181 (1981), 115. Wrede geht freilich davon aus, dass frühere Darstellungen des Genius populi Romani als bärtiger älterer Mann das Bild des genius Senatus vorweg nehmen.

38 Missere 1990, a.a.O. (Anm.9), 88-99.

39 Zur klassischen Bilderfindung des bärtigen Demos: Messerschmidt 2003, a.a.O. (Anm.9), 10-21; A.C. Smith, Polis and personification in Classical Athenian art (Leiden, Boston 2011), 96-102 (mit der älteren Lit.); zum Demos allgemein Lexicon Iconographicum Mythologiae Classicae III (München, Zürich 1986), 375-382 s.v. Demos (O. AlexandriTzahou).

40 So z.B. Alexandri-Tzahou 1986, a.a.O. (Anm.39), 382; s. auch die Zusammenstellung bei Missere 1990, a.a.O. (Anm.9), 89. Auch in der Plastik wird in der früheren Kaiserzeit der bärtige Demos-Typ beibehalten: vgl. die Darstellung auf dem Zoilos-Fries: R.R.R. Smith, The monument of C. Julius Zoilos. Aphrodisias I (Mainz 1993), 32 f. Taf. 10. 11. 
fen, wobei anscheinend von den Schöpfern der Personifikation die Diskrepanz zwischen römischen und griechischen Vorstellungen im Genus in Kauf genommen wurde. Die Wahl des Geschlechtes muss auf römische Terminologie zurückgehen, die Nutzung des griechischen Namens $\Sigma u ́ \gamma \chi \lambda \eta \tau \circ \varsigma$ beweist jedoch die Absicht, von griechischer Seite in das eigene Begriffssystem einzubinden und schließlich die Gottheit zu integrieren. Sowohl der Kult selbst als auch die Personifikation weisen jedoch eindeutig auf asymmetrische Machtverhältnisse zwischen Hauptstadt und Provinz hin.

Das aus dem Genuswechsel entstehende Problem besteht in der Rezeption dieser vergöttlichten Institution und ihrer vorgegebenen bildlichen Erscheinung in einer Gesellschaft, die sie - rein sprachlich - weiblich auffasst. ${ }^{41}$ Eine beabsichtigte Entschärfung des Gegensatzes ist vielleicht darin zu sehen, dass auf den Münzen Smyrnas zunächst als Beischrift auch das geschlechtsneutrale $\Theta E O \Sigma \Sigma$ YNK $\Lambda$ HTO $\Sigma$ verwendet wird. ${ }^{42}$ Spätestens seit der mittleren Kaiserzeit wird dann jedoch überwiegend IEPA ¿YNK $\Lambda$ HTO $\Sigma$ als Legende gebraucht, so dass die Diskrepanz zwischen Bild und Text offen zu Tage tritt. ${ }^{43}$ Die Ikonographie von Synkletos wird jedoch unverändert beibehalten, so bleibt auf den zahlreichen städtischen Prägungen Smyrnas das Bild der jugendlichen männlichen Senatsgottheit bis in das 3. Jh. hinein vorherrschend (Abb. 2), wobei sich freilich diese

41 Die Personifikation von Synkletos ist ein besonders eindeutiger Beleg für die gender-Problematik bei Personifikationen. Allgemein, vor allem aber zur Tatsache, dass im griechischen Bereich die meisten Verkörperungen weiblich sind: E.J. Stafford, 'Masculine values, feminine forms: on the gender of personified abstractions', in L. Foxhall, J. Salmon (eds.), Thinking men. Masculinity and self-representation in the classical tradition (London, New York 2006), 43-56; vgl. auch D. Burton, 'The gender of death', in E. Stafford, J. Herrin (eds.), Personification in the Greek world (London 2005), 45-68; für die römische Zeit liegt bisher keine ausführlichere Untersuchung vor, sondern man belässt es bisher bei eher vereinzelten Bemerkungen wie zum ‘männlichen' Aussehen der Gerousia: Schultz 1997, a.a.O. (Anm.10), 6o8. Anscheinend war die Charakterisierung bereits für die Römer nicht immer eindeutig: vgl. Serv., Aen. 2.351, genio urbis Romae sive mas sive femina. Ähnlich gelagert die Bennung von weiblichen Stadtpersonifikationen im Typus der 'Tyche von Antiochia', als Genius auf Münzen römischer Kolonien in Kleinasien, z.B. aus Kremna oder Apameia in Bithynien, sowie andere Frauenfiguren, so aus Antiochia ad Pisidiam oder Antiochia am Orontes (hierzu M. Meyer, Die Personifikation der Stadt Antiocheia. Ein neues Bild für eine neue Gottheit (33. Ergänzungsheft Jahrbuch des Deutschen Archäologischen Instituts) (Berlin 2006), 391-397 Appendices A-D. Frühester Beleg ist der Genius terrae Africae auf Denaren des Qu. Caecilius Metellus Scipio (48-46 v. Chr.) in Gestallt der löwenköpfigen Göttin Tanit/Caelestis: Lexicon iconographicum mythologiae classicae I (München, Zürich 1981), 255 s.v. Africa (M. Le Glay); Meyer a.a.O., 396 App. D 3.

42 Klose 1987, a.a.O. (Anm.23), $130 \mathrm{n}^{\circ}$ II 1-11 (pseudoautonom, neronisch), III 19-61; RPC $420 \mathrm{n}^{\mathrm{o}} 2481$ (pseudoautonom, nach-neronisch); Burnett, Amandry, Ripollès 1992, a.a.O. (Anm.23), $420 \mathrm{n}^{\circ} 2489$.

43 Klose 1987, a.a.O. (Anm.23), 147 no V 1-14 (pseudoautonom, traianisch) 


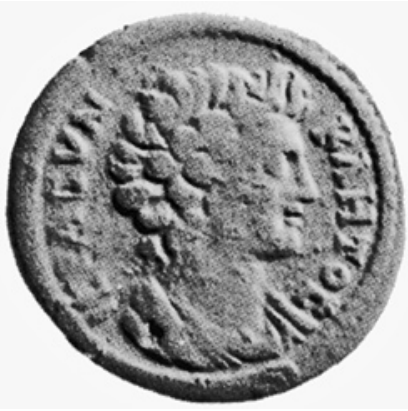

Abb. 2: AE Smyrna: Synkletos

idealen Jünglingsköpfe in Einzelheiten, z.B. der Länge der Haare, unterscheiden können. ${ }^{44}$ An der Tatsache, dass immer junge Männer wiedergegeben sind, ist jedoch nicht zu zweifeln. ${ }^{45}$ Engste Parallelen bestehen während der Kaiserzeit zu Münzen mit der Personifikation des Demos, die eine identische Büste eines jugendlichen, langhaarige Mannes zeigen, so dass grundsätzlich keine ikonographische Möglichkeit besteht, Synkletos und Demos zu unterscheiden. ${ }^{46}$ Dies ist prinzipiell nur möglich, wenn beide Personifikationen als Ganzfiguren wiedergegeben sind, da Demos ein Himation, Synkletos eine Toga trägt. Probleme gibt es bei der Unterscheidung der Personifikationen von Senat und römischem Volk in griechischer Ikonographie: Auf einem der Reliefs vom Sebasteion in Aphrodisias wird Claudius von einer jugendlich männlichen Personifikation bekränzt, bei der nicht eindeutig zu unterscheiden ist, ob es sich um den Demos der Römer oder Synkletos handelt. ${ }^{47}$

Neben Smyrna sind aus vielen kleinasiatischen Städten so genannte pseudo-autonome Münzen erhalten, die anstelle des Kaiserporträts

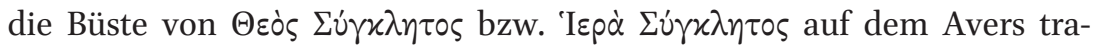
gen, wobei im Normalfall dieselbe Ikonographie wie in Smyrna verwen-

44 Klose 1987, a.a.O. (Anm.23) 184 f. no XIV 1-4; 186-188 no XV 1-10; 189 n $^{\circ}$ XVI 1; 189-192 $n^{\circ}$ XVIII 1-12; 194 f. no XIX 2-6; 196 f. no XX 2-6; 197-203 no XXI 1-44 bzw. $48 ; 327$ f. $\mathrm{n}^{\circ}$ LXXVIII 1-6.

45 Vor allem für die Frisur gibt es keine direkten Parallelen bei weiblichen Idealköpfen. Auffällig und für einen Mann ungewöhnlich ist das stoffreiche, aufwändig gefältelte Gewand, doch auch das ist kein Hinweis auf weibliche Tracht, sondern der Versuch des Stempelschneiders, den Stoffreichtum der Toga zum Ausdruck zu bringen und vom griechischen Himation abzusetzen.

46 So bereits Fears 1978, a.a.O. (Anm.6), 280.

47 R.R.R. Smith, 'The imperial reliefs from the Sebasteion at Aphrodisias', Journal of Roman Studies 77 (1987), 114 f. Taf. 12. 12, 3-4. 
det wurde. ${ }^{48}$ Es liegt somit nahe, in der Vielzahl ähnlicher jugendlicher Senatsgottheiten eine Orientierung an die smyrnäischen Prägungen und letztlich an dem offiziellen Provinzialkult zu vermuten, dessen unter Tiberius errichtetes Kultbild in der Münzprägung reflektiert wurde. Dies gilt sowohl für den 'Prägebezirk' Smyrna als auch weit darüber hinaus. ${ }^{49}$

Trotz dieser weitgehenden ikonographischen Übereinstimmung zeigt sich, dass das Bild des Senats nicht in allen Einzelheiten festgeschrieben und seine Verwendung nicht verbindlich war, so dass generell zwei unterschiedliche Optionen der Darstellung zu scheiden sind:

1.) Beibehaltung der Jünglingsbüste mit Variationen im Detail

2.) grundsätzlich neue Erscheinungsform

Zur ersten Option: Auch wenn der Senat als langhaariger junger Mann gezeigt wird, können die Bilder in Details voneinander abweichen. So kann die Senatspersonifikation auch einen Lorbeerkranz ${ }^{50}$ oder ein Diadem $^{51}$ tragen. Anhand dieser Synkletos-Köpfe mit Diadem wurde versucht, direkte hellenistische Vorläufer zu postulieren; ${ }^{52}$ eine solche Ableitung von Diadochen-Porträts ist jedoch schon aufgrund fehlender hellenistischer Belege für Synkletos nicht zu beweisen. Zudem zeigen die Münzen aus Smyrna, die das gemeinsame Urbild wiedergeben, keine Büste mit Königsbinde. Wesentlich wahrscheinlicher ist es deshalb anzunehmen, dass das Diadem eine spätere Zutat ist, so dass ein traditionelles Zeichen der Autorität auf ein neues Bild übertragen wurde. Grundsätzlich ist Synkletos wesentlich häufiger barhäuptig dargestellt als sein ikonographisches Gegenstück, der griechische Demos. ${ }^{53}$

48 S. Anhang I.

49 Zu den kleinasiatischen Prägebezirken: K. Kraft, Das System der kaiserzeitlichen Münzprägung in Kleinasien (Berlin 1972); zum Prägebezirk Smyrna: Klose 1987, a.a.O. (Anm.23), 86-91. Die Stempelkopplungen anhand von pseudoautonomen SynkletosPrägungen exemplifiziert durch Harl 1987, a.a.O. (Anm.16), Taf. 6, 4-8.

50 z.B. Aphrodisias (gordianisch): M. Spoerri Butcher, Roman provincial coinage VII 1: Province d'Asie (Paris, London 2006): 241 f. no 629-631; Klazomenai (69-79): A. Burnett, M. Amandry, I. Carradice, Roman provincial coinage II: From Vespasian to Domitian (AD 69-96) (London, Paris 1999), $160 \mathrm{n}^{\circ} 1033,1-4$.

51 z.B. Phokaia (Ionia) (235-238): I.A. Carradice, Sylloge Nummorum Graecorum VI: The Lewis Collection in Corpus Christi College Cambridge. Part II: The Greek Imperial coins (Oxford 1992) nº 1440 Taf. 31 1440(1); Aphrodisias: B.V. Head, Catalogue of the Greek coins of Caria, Cos, Rhodes, \& C. (London 1897), 30 f. n 29-32 Taf. V 13.

52 Forni 1953, a.a.O. (Anm.2) 62 f.; K. Harl, Political attitudes of Rome's eastern provinces in the third century A.D. (Yale 1978), 282; Missere 1990, a.a.O. (Anm.9), 98 Anm.171.

53 Vgl. die Zusammenstellung bei Missere 1990, a.a.O. (Anm.9), 89. 
Zur zweiten Option: Interessanter als diese Variationen eines Urbildes sind freilich die Fälle, in denen für Synkletos eine ganz andere, vom Erscheinungsbild der Kultstatue in Smyrna grundsätzlich abweichende Ikonographie gewählt wurde. Aufschlussreich sind sie vor allem deswegen, weil sie zeigen, dass die Ikonographie der Senatsgottheit nicht zentral von Rom vorgeschrieben war und dass die Entscheidung, welches Bild verwendet wurde, auf Seiten der prägenden Poleis, also der provinzialen Führungsschicht lag.

Vergleichsweise häufig sind Münzen, die dem griechischen Sprachgebrauch entsprechend die Büste einer Frau mit oder ohne Schleier zeigen. ${ }^{54}$ Eine Besonderheit bietet eine Prägung des 2. oder 3. Jhs. aus Perperene in Mysien (Abb. 3): ${ }^{55}$ Hier trägt Synkletos eine Mauerkrone ganz in Tradition einer hellenistischen Stadtgottheit, wie sie mit der so genannten Tyche von Antiochia beispielhaft konzipiert wurde. ${ }^{56}$ In all diesen Fällen zeigt sich in der Ikonographie die Abhängigkeit von der griechischen Boulê. Auf Münzen ist die Ratsversammlung nämlich meist ebenso in Form eines Frauenkopfes wiedergegeben, wobei Boulai wie auch Synkletoi selten mit Mauerkrone auftreten. ${ }^{57}$ Auch hierin folgt also die Senatsgottheit der lokalen städtischen Form der Ratsversammlung. Unterschiede bestehen vor allem darin, dass weibliche Synkletoi anscheinend viel seltener Diadem oder Kranz tragen wie dies die Hierai Boulai, vor allem seit der Regierungszeit Caracallas tun. ${ }^{58}$

Eine Besonderheit sind die Münzen, die Synkletos auch mit ikonographischen Mitteln mit dem Kaiserhaus in Bezug setzen. Am einfachsten nachzuvollziehen ist dies im Fall der Prägungen aus Apollonis, die zur Legende IEPA $\Sigma$ YNK $\Lambda$ HTO $\Sigma$ eine weibliche Büste mit typischem flavischen Löckchentoupet zeigen, ${ }^{59}$ so dass deshalb m. E. zu Recht eine Verbindung

54 Darstellungen einer weiblichen Senatsgottheit sind jedoch deutlich seltener als die der jugendlich-männlichen, da viele der von Forni 1953, a.a.O. (Anm.2) und auch noch von Kienast 1985, a.a.O. (Anm.2) als weiblich angesprochenen Senatsdarstellungen auf Münzen nur Varianten des normalen Typus sind.

55 F. Imhoof-Blumer, Kleinasiatische Münzen (Wien 1901), 32 s.v. Perperene Nt. 1; Forni 1953, a.a.O. (Anm.2), $87 \mathrm{n}^{\circ} 22$ Taf II 16; Kienast 1985, a.a.O. (Anm.2), 267 f. Anm.65; M.B. Barth, J. Stauber, 'Die Münzen von Perperene', Epigraphica anatolica 23 (1994) 63 f. nº 17

56 Meyer 2006, a.a.O. (Anm.41), vor allem 175-178; zur Mauerkrone: ebenda, 110-112, 157-164.

57 Vgl. die Tabelle bei Missere 1990, a.a.O. (Anm.9), 101.

58 Ebenda $77 \mathrm{f}$.

59 Burnett, Amandry, Carradice 1999, a.a.O. (Anm.5o), 148 n $^{\circ}$ 95o, 1-8; 951, 1-10; 952, 1-3. 


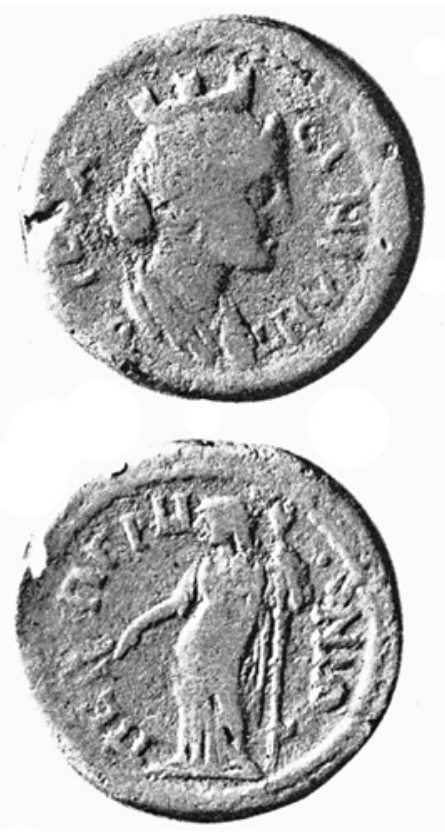

Abb. 3: AE Perperene: Büste der Synkletos mit Mauerkrone; Demeter

zu Domitia oder Iulia Titi gezogen wurde. ${ }^{60}$ Schwieriger zu belegen ist die Verknüpfung von Kaiserporträt und jugendlichem Normaltyp der Personifikation, doch erscheinen die physiognomischen Ähnlichkeiten zwischen Nero und Synkletos auf Münzen aus Sardes ${ }^{61}$ und zwischen Vespasian und Synkletos aus Kyme ${ }^{62}$ überzeugend. Die Personifikation des Senats konnte folglich auf verschiedene Weise 'belebt' werden konnte. In diesen Fällen sollte durch eine Angleichung an Bildnisse des Kaisers und seine Familie der enge Bezug zum Herrscherhaus und somit zu Rom zum Ausdruck gebracht werden, ist der Kult des Senats doch im Kontext des Kaiserkultes in Smyrna etabliert worden. ${ }^{63}$

60 So bereits Forni 1953, a.a.O. (Anm.2), 61; vgl. jetzt auch U. Hahn, Die Frauen des römischen Kaiserhauses und ihre Ehrungen im griechischen Osten anhand epigraphischer und numismatischer Zeugnisse von Livia zu Sabina (Saarbrücken 1994), 241, 359 n 246-247.

61 Burnett, Amandry, Ripollès 1992, a.a.O. (Anm.23), 490 nº 3008, 1-19.

62 Burnett, Amandry, Ripollès 1992, a.a.O. (Anm.23), $15^{2} \mathrm{n}^{\circ}$ 971, 1-9.

63 Zum Phänomen der Angleichung auch über Geschlechtergrenzen hinaus: R. Varner, 'Transcending Gender: Assimilation, Identity and Roman Imperial Portraits', in S. Bell, I. Hansen (eds.), Role Models: Identity and Assimilation in the Roman World (Ann Arbor 2008), 185-205. 


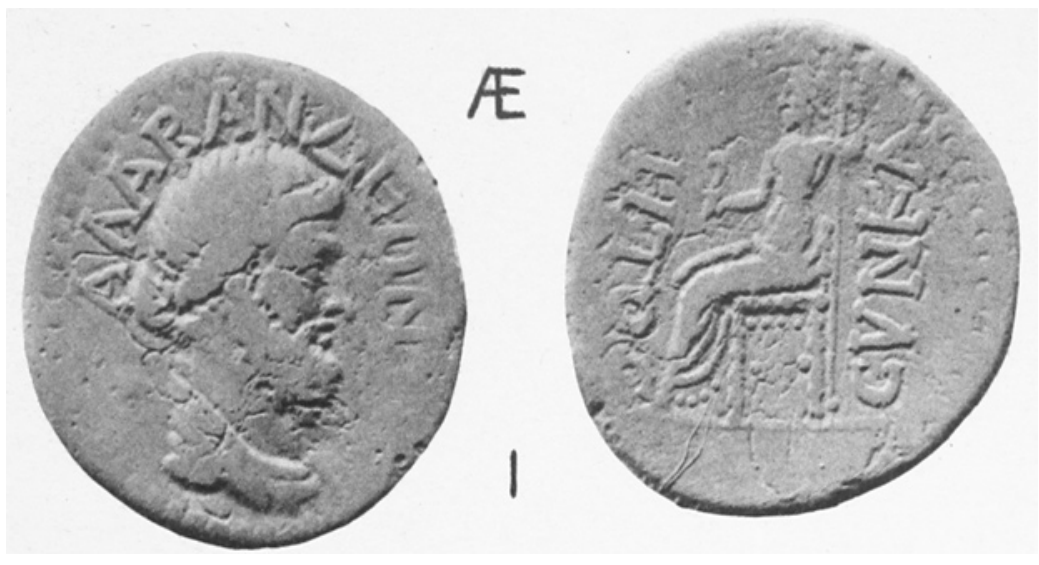

Abb. 4: AE Alabanda: Demos, Synkletos thronend

An einer Münze aus Alabanda lässt sich eine andere Option aufzeigen (Abb. 4), wie Synkletos als Gottheit 'aufgeladen' wurde:64 Dargestellt ist die Personifikation als ganzfigurige Sitzstatue, im Vergleich zu den vielen Büsten eine eher unübliche Form der Wiedergabe. Die - wohl weibliche Senatsgottheit sitzt auf einem Thron und hält in der erhobenen linken Hand ein langes Stabzepter, in der ausgestreckten Rechten einen oben eingekrümmten Stab, einen lituus. Das Sitzmotiv an sich und das Szepter erinnern eindeutig an thronende Gottheiten, den Bewohnern in Alabanda bekannt vor allem von gleichzeitigen Münzbildern des thronenden Zeus. ${ }^{65}$ Anders verhält es sich mit dem lituus, der etwas spezifisch Römisches ist: Bekannterweise ist der Krummstab Signet der Auguren, doch kann er auch Insignie der Befehlsgewalt, des imperium, und somit im wahrsten Sinne des Wortes ein imperiales Zeichen sein. ${ }^{66}$ In diesen Kontext gehört die Darstellung des Augustus auf der Gemma Augustea mit lituus. ${ }^{67}$ In Alabanda selbst ist Vespasian mit einem Krummstab auf einer Münze wiedergegeben. ${ }^{68}$ Die Verbindung Synkletos - lituus kommt bereits früher

64 Burnett, Amandry, Ripollès 1992, a.a.O. (Anm.23), $183 \mathrm{n}^{\circ}$ 1203, 1; vgl. auch Canciani 1994, a.a.O. (Anm.12), $828 \mathrm{n}^{\circ} 5$.

65 Burnett, Amandry, Ripollès 1992, a.a.O. (Anm.23), 183 n $^{\circ} 1202,1$.

66 Siebert 1999, a.a.O. (Anm.26), 130-132.

67 E. Zwierlein-Diehl, Magie der Steine. Die antiken Prunkkameen im Kunsthistorischen Museum (Wien 2008), 98-123; zum lituus: ebenda 101 f., 116 Abb. 40.

68 Es ist dies die Vorderseite der Prägung mit dem thronenden Zeus (hier Anm.59). 
auf Münzen vor, so in iulisch-claudischer Zeit in Pergamon, Metropolis oder Akmoneia, um nur einige Beispiele zu nennen. ${ }^{69}$

Die Prägung aus Alabanda zeigt somit exemplarisch zwei unterschiedliche Optionen, den Senat mit besonderer Befehlsgewalt darzustellen: zum einen durch Angleichung an übliche griechische Götter-Ikonographie, zum anderen durch Anleihen an die offizielle Bildersprache des römischen Herrscherkultes.

Insgesamt lassen sich somit in der kleinasiatischen Münzprägung verschiedene Mittel unterscheiden, die Senatsgottheit ikonographisch als Gottheit zu konstituieren und als entsprechend machtvoll ins Bild zu setzen:

- Herstellung eines Bezugs zum Kaiserhaus in Form der Bildnisangleichung

- Übernahme von Elementen der offiziellen römischen Bildersprache

- Übernahme von Elementen der griechischen Götterikonographie

- Übernahme von Elementen der hellenistischen Herrscherikonographie

Die mit Abstand meisten der eben betrachteten Sonderfälle der SynkletosIkonographie stammen aus dem 1. Jh. n. Chr., seit dem 2. Jh. folgt die überwiegende Anzahl der Darstellungen dem jugendlichen Erscheinungsbild des Prototyps in Smyrna mit nur geringen Variationen. Innerhalb einer Zeitspanne von ungefähr 100 Jahren erfolgte eine Integration nicht nur des provinzialen Kultes, sondern auch die Übernahme der durch die Kultstatue in Smyrna fixierten Ikonographie in einen Großteil der asiatischen Städte.

Darin wird deutlich, wie sich nach anfänglicher Experimentierfreudigkeit eine homogenere Vorstellung vom Synkletos unter Verwendung des für den Provinzialkult in Smyrna konzipierten Urbilds als Gegenstück zum Demos und zur Boulê durchsetzte. Konsequente Einheitlichkeit z. B. als Folge zentralen Transports und einer offiziell geregelten Verbreitung von Kultikonographie ist jedoch nicht festzustellen.

\section{Integration von Synkletos in die städtischen Panthea Kleinasiens}

$\mathrm{Zu}$ fragen ist nun, welchen Stellenwert die Senatspersonifikation als Gottheit, aber auch als römische Institution im kaiserzeitlichen Kleinasien

69 Burnett, Amandry, Ripollès 1992, a.a.O. (Anm.23), 404 no 2376, 1-6; ähnlich Münze aus Akmoneia: ibid., $513 \mathrm{n}^{\circ} 3167,1-5$; aus Metropolis: ibid., 426 f. n ${ }^{\circ}$ 2524-2525 usw. 
besaß. Die große Bedeutung wird zum einen dadurch sichtbar, dass Synkletos auf Münzen an Stelle des Kaisers treten kann. Bei den bisher betrachteten Münzen handelt es sich meist um so genannte pseudoautonome Prägungen, die so bezeichnet werden, weil kein Kaiserbild auf die Vorderseite der Münzen gesetzt wurde, so dass der Anschein einer unabhängigen Stadtprägung entstehen kann. ${ }^{70}$

Grundsätzlich lässt sich bei einer übergreifenden Untersuchung der Rückseiten der Synkletos-Münzen feststellen, dass im Motivrepertoire keine Kombinationen besonders häufig vorkommen und sich die Prägungen nicht vom Rest der anderen 'Greek Imperials' unterscheiden. ${ }^{71}$ Mit großem Abstand werden zusammen mit Synkletos herkömmliche Gottheiten des lokalen Pantheon dargestellt. Neben Athena, Zeus, und anderen olympischen Göttern werden auch Kybele, Sarapis und Men bedacht. Synkletos tritt zudem besonders häufig zusammen mit der ephesischen Artemis auf. Diese Vergesellschaftung ist ein Indiz dafür, dass Artemis eben nicht als Zeichen des offenen oder verdeckten Widerstandes gegen Rom interpretiert werden kann, wie dies vor kurzem von J. Elsner behauptet wurde. ${ }^{72}$ Artemis-Verehrung und Verehrung römischer Instanzen sind tatsächlich nur zwei Seiten einer Medaille. Annähernd gleich häufig sind Personifikationen mit Synkletos verbunden, insbesondere die entsprechende Stadtgöttin bzw. Tyche oder Homonoia als Ausdruck besonderer Städtepartnerschaften. ${ }^{73}$ Es folgen in quantitativer Reihung Szenen aus dem lokalen Mythos bzw. Heroen mit dezidiertem Ortsbezug und erst anschließend spezifisch 'römische' Motive wie der Kaiser oder römische Personifikationen, d. h. Roma oder der Demos der Römer. SynkletosMünzen zeigen folglich kein besonderes 'römisches' Spektrum und auch keine abweichende Mengenverteilung, sondern spiegeln das 'normale' Repertoire kleinasiatischer Münzen wider. Die Senatsgottheit wird also in das kleinasiatische religiöse System integriert bzw. ist ein integrales

$70 \mathrm{Zu}$ 'pseudoautonomen' Prägungen: A. Johnston, “The so-called "pseudo-autonomous" Greek imperials', American Numismatic Society Museum Notes 30 (1985), 89-112.

71 Die Angaben beruhen auf einer Suche mit Hilfe der Datenbank „Projekt ISEGRIM Datenbank kleinasiatischer Münzen" des Lehrstuhls für Alte Geschichte der HeinrichHeine-Universität Düsseldorf (Stand 2004); zu Rate gezogen auch: P.R. Franke, W. Leschhorn, A.U. Stylow, Sylloge Nummorum Graecorum Deutschland, Sammlung von Aulock. Index (Berlin 1981).

72 J. Elsner, 'The origins of the icon: pilgrimage, religion and visual culture in the Roman East as "resistance" to the centre', in S. Alcock (ed.), The early Roman Empire in the East (Oxford 1997), 178-199, vor allem 188-191.

73 Zu Homonoia-Prägungen: P.R. Franke, M.K. Nollé, Die Homonoia-Prägungen Kleinasiens und der thrakischen Randgebiete (Saarbrücken 1997). 
Bestandteil dieses Systems, da sie auch einen Anteil an der Ausbildung dieses Systems hat.

Synkletos als Teil städtischer Kultpraxis:

Die Stiftung des Salutaris in Ephesos

Bestätigt wird dieses Ergebnis durch einen Beleg aus einem anderen Kontext, der Stiftung des C. Vibius Salutaris aus trajanischer Zeit in Ephesos: ${ }^{74}$ Salutaris stiftet $3_{1}^{1}$ Statuen aus Edelmetall, die in einer großen Prozession durch die Stadt getragen werden sollen. ${ }^{75} \mathrm{Zu}$ diesen Skulpturen gehören neun Artemis-Statuen, ein Trajan- und ein Plotina-Porträt, Statuen der Stadtgründer Androklos und Lysimachos und zahlreiche Personifikationen, so die Boulê von Ephesos, die Gerousia, der Ritterstand, die Ephebeia, Darstellungen der Phylen, der Demos der Römer und eben der Senat. ${ }^{76}$ Auch wenn es sich bei dem Stifter um einen römischen Ritter gehandelt hat, so wird hier dieselbe enge Verbindung von lokaler Hauptgottheit, den örtlichen politischen Instanzen, Akteuren der lokalen Geschichte und des lokalen Mythos, dem Kaiserhaus und Repräsentanten Roms zum Ausdruck gebracht, wie sie typisch für die Münzprägung ist. Der Stellenwert des Senats innerhalb dieses Kultfestes kann sogar noch weiter bestimmt werden: Zum einen wird er nach Artemis und dem regierenden Kaiser und seiner Frau an dritter bzw. vierter Stelle genannt und bildet zusammen mit der Personifikation des lokalen Rates ein Paar. Zum anderen weist die Gewichtsangabe von 4 Pfund und zwei Unzen hin auf eine Position zwischen den Statuen der Götter, insbesondere Artemis, der allein auch eine Goldstatue zugedacht war, und den übrigen Personifikationen (Anhang II). Das Gewicht der Statuen für Trajan und Augustus ist unbekannt, sicher ist jedoch, dass das Porträt Plotinas leichter war als die Statue von Synkletos. Auch wenn hier der Unterschied darin liegen kann, dass diese eine ganzfigurige Darstellung war, jene jedoch eine Büste, so wird dennoch deutlich, dass die Senatsgottheit zwar nicht die wichtigste Stellung, aber doch eine prominente und auffällige Position innerhalb der Statuenprozession einnahm. Wie bei den Münzen zeigt sich die aktive

74 Grundlegend: Rogers 1991, a.a.O. (Anm.16); zuletzt F. Graf, 'Ritual restoration and innovation in the Greek cities of the Roman Imperium', in A. Chaniotis (ed.), Ritual dynamics in the Ancient Mediterranean (Stuttgart 2011), 105-117 (mit der älteren Lit.).

75 Übersetzung der Inschrift: Rogers 1991, a.a.O. (Anm.16), 152-185.

76 Zur Statue des Senats: Rogers 1991, a.a.O. (Anm.16), $91 \mathrm{f}$. 
Einbindung in ein lebendiges Kultsystem, die nicht darauf hinweist, dass die Verehrung des Senats eine bloße Pflichtübung gegenüber Rom war. Interessant in mehrfacher Hinsicht ist, dass Synkletos zusammen mit der Personifikation der Boulê genannt wird und nicht mit dem Demos der Römer: Diese Zusammenstellung zeigt erstens, dass man nicht nach römischem Vorbild agierte, sondern eigene Vorstellungen umsetzte, da in der Hauptstadt Genius Senatus und Genius Populi Romani regelhaft kombiniert wurden. Zweitens wird auch klar, dass die römischen Instanzen nicht als einheitlicher (Fremd-)Körper aufgenommen wurden, sondern dass man sie mit lokalen Einrichtungen zusammennahm. Drittens ist auffällig, dass anscheinend immer Frau-Mann-Paare miteinander verbunden werden, vorausgesetzt, dass auch hier der Senat als männliche Personifikation auftritt, so dass für die ephesische Prozession eine Darstellung von Synkletos in Gestalt eines jungen Mannes vermutet werden kann.

\section{Ergebnis}

Für die Integration des Senats war der römische Kaiser Ausgangspunkt, da nach Tacitus Tiberius ihn als Kultempfänger in Smyrna bestimmte. In kaiserlichem Auftrag wurde auch eine bildliche Umsetzung der römischen Institution geschaffen, die weder griechischen Vorstellungen noch der - späteren - römischen Visualisierung folgte. Dennoch entsprach die Verkörperung der Senatsgottheit dem grammatikalischen Geschlecht des lateinischen Terminus senatus und somit eher der römischen Auffassung. Dies wurde jedoch erst evident dadurch, dass man das griechische $\Sigma$ v́ $x \lambda \eta \tau \circ \varsigma$ als Kultbezeichnung wählte, im Rahmen griechischer Begrifflichkeit blieb und ein - gräzisiertes - Fremdwort vermied. Fakt ist aber auch, dass es keine Anknüpfung an bereits bestehende Ikonographien von Boulê oder auch Gerousia gab. Die Aneignung des Kultbildes auf provinzweiter Ebene erfolgte durch die Poleis, wobei die lokalen Eliten zunächst die treibende Kraft gewesen sein müssen. ${ }^{77}$ Die Integration erweist sich als länger dauernder und mehrsträngiger Prozess, wie sich anhand der Bildfassungen von Synkletos ablesen lässt, wo verschiedene Möglichkeiten

77 Zur Rolle der Eliten in der Münzprägung exemplarisch: B. Weisser, 'Pergamum as paradigm', in C. Howgego, V. Heuchert, A. Burnett (eds.), Coinage and identity in the Roman provinces (Oxford 2005), 135-142; grundlegend, aber für eine andere Provinz: K. Butcher, 'Information, legitimation, or self-legitimation? Popular and elite designs on the coin types of Syria', in ebenda, 143-156. 
zur Verbesserung und Erleichterung der Akzeptanz wie Femininisierung bzw. Angleichung an Kaiserbildnisse erprobt (und im Laufe der Zeit überwiegend wieder verworfen) werden. Als entscheidend erweist sich die provinziale Struktur, die Kulttradition und wohl auch die kaiserliche Autorität bzw. Legitimierung des Kultbildes im Tempel von Smyrna. Als Gottheit werden der Senat und sein Bild schließlich integraler Bestandteil der städtischen Panthea wie Einheitlichkeit und Beständigkeit der Ikonographie bezeugen. Selbst der Widerspruch zwischen grammatikalischem Geschlecht und Visualisierung wird nicht als so gravierend angesehen, dass er unbedingt aufzulösen wäre. Synkletos erweist sich dadurch auch als eine Gottheit mit eigenem griechischem Charakter, was wiederum dem Bild des Kaiserkultes in Kleinasien gleicht. Es wäre jedoch ein Fehler, nur die griechische Komponente zu betonen und in Synkletos nur eine Gottheit zu sehen, die zwar anfangs etwas mit Rom zu tun hatte, später aber dann so perfekt integriert war, dass sie ein vollständig kleinasiatischeinheimisches Eigenleben entwickelte. Ein Blick auf die Verbreitung der Zeugnisse für die Senatsgottheit, im Wesentlichen von Münzen und Inschriften, in Kleinasien belegen die römische Kondition: Mit nur wenigen Ausnahmen, z.B. aus Mallos in Kilikien oder Side in Pamphylien, bleibt die Verehrung der Senatsgottheit auf die Provinz Asia beschränkt. ${ }^{78}$ $\mathrm{Zu}$ erklären ist dieses Muster durch die provinzial gebundene Organisation des Kultes und wiederum deren Abhängigkeit von politischen Verwaltungsgrenzen die von Rom festgelegt wurden. Bei aller Beliebtheit in Asia, Synkletos wird nicht exportiert und besitzt kein wirkliches Eigenleben jenseits der Grenzen. Trotz aller Eigenständigkeit und der gelungenen Integration in ein griechisches religiöses System wird doch deutlich, dass es sich hier um ein provinziales religiöses System im wörtlichen Sinne einer Provinzialreligion handelt, das ohne Rom nicht funktionieren kann. ${ }^{79}$

78 J. und L. Robert, Bulletin épigraphique (1954), 112; Forni 1953, a.a.O. (Anm.2), 11-14: 99 Städten, die Münzen mit Synkletos prägen, stehen drei in Creta et Cyrenaica (einer anderen senatorischen Provinz), drei in Cilicia und einer in Pisidia gegenüber.

79 Zum Begriff der Provinzialreligion: J. Rüpke, 'Römische Religion und „Reichsreligion“: Begriffsgeschichtliche und methodische Bemerkungen', in H. Cancik, J. Rüpke (eds.), Römische Reichsreligion und Provinzialreligion (Tübingen 1997), 3-23; zum Thema: M. Beard, J. North, S. Price, Religions of Rome I: A History (Cambridge 1998), 313-363; C. Ando, 'Exporting Roman religion', in J. Rüpke (ed.), A companion to Roman religion (Malden, MA 2007), 429-445. 
Anhang I: Liste der Städte der Provinz Asia, die pseudoautonome Münzen mit der jugendlichen, langhaarigen Synkletos-Personifikation prägen ${ }^{80}$

Verwendete Abkürzungen:

Aulock, Phrygien: H. von Aulock, Münzen und Städte Phrygiens (Tübingen 1980/1987).

BMC: H. Mattingly, Coins oft he Roman Empire in the British Museum (London 1923-1950).

Fitzwilliam: S.W. Grose, Catalogue of the MacClean Collection of Greek coins. Fitzwilliam Museum, Cambridge vol. 3 (Cambridge 1929).

Fritze, Mysien: H. von Fritze, Die antiken Münzen Mysiens (Berlin 1913)

Imhoof, GM: F. Imhoof-Blumer, Griechische Münzen (München 189o).

Imhoof, KM: F. Imhoof-Blumer, Kleinasiatische Münzen (Wien 1901/02).

Imhoof, LS: F. Imhoof-Blumer, Lydische Stadtmünzen (Genf 1897).

Klose, Smyrna: D. Klose, Die Münzprägung von Smyrna in der römischen Kaiserzeit (Berlin 1987).

RPC I: A. Burnett, M. Amandry, P.P. Ripollès, Roman provincial Coinage I: From the death of Caesar to the death of Vitellius (London 1992)

Slg. Lindgren: H.C. Lindgren, Ancient bronze coins of Asia Minor and the Levant from the Lindgren collection (San Mateo/CA 1985).

Slg. Weber: L. Forrer, The Weber Collection vol. 3: Asia minor (London 1925-1929)

SNG: Sylloge nummorum Graecorum

Aiolis:

Aigai (177-192; 193-211): Slg. Lindgren I 387; SNG Kopenhagen XXI 17

Elaia (63-68; 238-244): RPC I 2409; Slg.Weber 5557

Kyme (70-73; 117-192; 238-244; 253-268): SNG Slg. von Aulock 1645; SNG Tübingen 2703; SNG Kopenhagen XXI 126; SNG Kopenhagen XXI 128(1)

Myrina (Kaiserzeit): SNG Kopenhagen XXI 227

Temnos (200-250 bzw. 218-222; 222-235; 238-244; 244-249; 253-268): SNG Kopenhagen XXI 266; SNG Kopenhagen XXI 267; SNG Kopenhagen XXI 270; SNG Slg. Righetti (Bern) 0825/ SNG Kopenhagen XXI 272; SNG Slg. von Aulock 1676

80 Der Katalog erhebt keinen Anspruch auf Vollständigkeit und ist vor allem gedacht als Überblick über lokale und chronologische Verteilung der Prägungen und als Hilfsmittel zur Auffindung von Abbildungen. 


\section{Ionien:}

Erythrai (161-180; 244-249 bzw. 260; Kaiserzeit): SNG Kopenhagen XXIII 749; SNG Kopenhagen XXIII 751; SNG Kopenhagen XXIII 779; BMC XIV 143 Nr.230

Klazomenai 69-79; (Kaiserzeit): SNG Kopenhagen XXII 110; SNG Kopenhagen XXII 111

Kolophon (238-249): Imhoof, KM 72 Nr.16

Magnesia am Mäander (200-276): SNG Kopenhagen XXIII 858

Phokaia (222-235; 235-238; 238-244; 244-249; Kaiserzeit): SNG Kopenhagen XXIII 1051; SNG Slg. von Aulock 2139; SNG Kopenhagen XXIII 1055; SNG Slg. Lewis (Cambridge) 1442; BMC XIV 218 Nr.116

Smyrna (54-68; 68-70; 100-105; 161-169; 182-185; 211-260; 218-222; 222235; 235-244; 238-240; 238 bzw. 241-249; 239-241; 242-244; 244-249 bzw. 255): RPC I 2481; RPC I 2489; SNG Kopenhagen XXIV 1253; SNG Kopenhagen XXIV 1299; SNG Kopenhagen XXIV 1303; SNG Kopenhagen XXIV 1323; Klose, Smyrna Nr. XVI; Klose, Smyrna Nr. XVIII; SNG Tübingen 3243; SNG Kopenhagen XXIV 1322; SNG Kopenhagen XXIV 1325; Klose, Smyrna Nr. XVIII; SNG Kopenhagen XXIV 1314; SNG Kopenhagen XXIV 1318; SNG Tübingen 3205; SNG Kopenhagen XXIV 1326

Teos (Kaiserzeit): SNG Kopenhagen 24, 1492

\section{Karien:}

Antiocheia (238-268; Kaiserzeit): SNG Kopenhagen XXV 42; Fitzwilliam 4671

Aphrodisias (100-276; 161-180; 193-211; 238-244; 250-276; 253-268; Kaiserzeit): SNG Slg. von Aulock 8o61; SNG Kopenhagen XXV 98; SNG Kopenhagen XXV 99; SNG Kopenhagen XXV 104; SNG Slg. von Aulock 8062; Fitzwilliam 4680; SNG Kopenhagen XXV 95

Apollonia (100-150; 161-180; 193-211): J.C. Milne, Numismatic Chronicle 1938, 257; SNG Slg. von Aulock 2481; SNG Slg. von Aulock 8068

Antiocheia (238-268; Kaiserzeit): SNG Slg. Righetti (Bern) o939; SNG Kopenhagen XXV 41

Attuda (180-220; 177-192; 193-211; Kaiserzeit): SNG Kopenhagen XXV 166; SNG Kopenhagen XXV 167; SNG Slg. Lewis (Cambridge) 1648; Imhoof, GM $87 \mathrm{Nr} .3$

Herakleia (161-169; 180-220; 193-211; Kaiserzeit): Imhoof, KM 134 Nr.9; SNG Slg. von Aulock 2544; SNG Slg. Lewis (Cambridge) 1656; SNG Kopenhagen XXV 394

Kidramos (Kaiserzeit): BMC XV 81 Nr.1 
Neapolis (69-96): SNG Kopenhagen XXV 451

Orthosia (180-220; Kaiserzeit): SNG Slg. von Aulock 2646; SNG Kopenhagen XXV $45^{8}$

Sebastopolis (100-276; 200-230; Kaiserzeit): BMC XV 146 Nr.4; SNG Kopenhagen XXVI 462; SNG Kopenhagen XXVI 463

Trapezopolis (180-220): SNG Slg. von Aulock 2738

Lydien:

Akrasos (193-211): BMC XVI 9 Nr.3

Aninetos (Kaiserzeit): Imhoof, KM 168 Nr.1

Apollonis (Kaiserzeit): SNG Kopenhagen XXVII 25

Apollonnoshieron (14-10o bzw. 217; 198-217): BMC XVI XXIII Nr. 4; BMC XVI 23 Nr.5; SNG Slg. Righetti (Bern) 1006

Attaleia (100-276; Kaiserzeit): SNG Kopenhagen XXVII 34(1); SNG Slg. von Aulock 2909

Bageis (98-117; 117-138; 161-192; 193-235; 200-268): SNG Slg. von Aulock 2913; BMC XV 31 Nr.4; Slg. Lindgren III 552A; SNG Kopenhagen XXVII 45; SNG Kopenhagen XXVII 44

Blaundos (100-276; 161-180; 251-253): SNG Kopenhagen XXVII 83; SNG Slg. Lewis (Cambridge) 1521; SNG Slg. von Aulock 2922

Daldis (69-96; 193-211; 198-268; Kaiserzeit): SNG Kopenhagen XXVII 110; BMC XVI 70 Nr.6; Slg. Lindgren III 456A; SNG Slg. von Aulock 1647(1)

Dioshieron (100-200; 138-192 bzw. 268): Imhoof, LS 65 Nr.7; SNG Slg. Righetti (Bern) 1022(1)

Gordos Iulia (117 bzw. 177-192): SNG Slg. von Aulock 2980; BMC XVI 90 Nr.5

Hermokapelia (117-138; 193-211): SNG Kopenhagen XXVII 165; BMC XVI $100 \mathrm{Nr} .13$

Hierokaisareia (54-138; 138 bzw. 177-192): SNG Slg. von Aulock 2957; Imhoof, LS 19 Nr.40

Hypaipa (Kaiserzeit): IMHOOF RSN 1923 S299,312

Hyrkanis (Kaiserzeit): BMC XVI 123 Nr.10

Kilbanioi (82-96): SNG Slg. Righetti (Bern) 1017(1)

Maionia (98-117; 161-180; 193-217; 249-251): Slg. Lindgren III 483; SNG Kopenhagen XXVII 219; SNG Slg. von Aulock 3014; SNG Kopenhagen XXVII 228

Magnesia (-27 bzw. 17-37; 138-268; 238-244): RPC I 2453; RPC I 2452A-C; SNG Kopenhagen XXVII 245; SNG Kopenhagen XXVII 249 
Mosteni (69-79; 117-192): Imhoof, KM 177 Nr.1; SNG Slg. Lewis (Cambridge) 1435

Nakrasa (98-161): SNG Slg. von Aulock 3034

Nysa (200-276): SNG Kopenhagen XXVII 311

Philadelpheia (98-117; 161-180; 193-211 bzw. 250; 198-268): Slg. Lindgren III 496; SNG Kopenhagen XXVII 354; BMC XVI 190 Nr.26; SNG Slg. von Aulock 3063; SNG Slg. von Aulock 3066

Saitta (193-268): SNG Slg. von Aulock 3091

Sala (98-117 bzw. 161; 138-161; Kaiserzeit): SNG Kopenhagen XXVIII 421; Slg. Lindgren III 512A; SNG Slg. von Aulock 3110; Imhoof, LS 132 Nr.3

Sardes (54-68; ca. 65; 70-73; 198-244): SNG Kopenhagen XXVIII 506; RPC I 3008; SNG Tübingen 3801; SNG Slg. Righetti (Bern) 1081

Silandos (98-117; 161-169 bzw. 192): Imhoof, LS 143 Nr.1; SNG Slg. von Aulock 3168; BMC XVI 278 Nr.3

Stratonikeia (98-138): SNG Kopenhagen XXVIII 555

Tabala (190-220): SNG Slg. von Aulock 8270

Thyateira (98-138; 100-200; 161-192; 198-217; 222-235; 235-238; Kaiserzeit): SNG Tübingen 3845(1); SNG Slg. Righetti (Bern) 1098; BMC XVI 296 Nr.28; Imhoof, LS 158 Nr.26; SNG Slg. von Aulock 3210(1); IMHOOF RSN 1923 S308,337; SNG Kopenhagen XXVIII 589

Tmolos (69-161): Imhoof, LS 164 Nr.2

Tomaris (Kaiserzeit): Imhoof, LS 165 Nr.3

Tralleis (161-276; 253-26o): BMC XVI 342 Nr.104; Imhoof, KM 187 Nr.7 Tripolis (98-117; 100-200; 138-192; 193-268 bzw. 200-276): SNG Slg. von Aulock 8295; SNG Kopenhagen XXVIII 721; SNG Slg. von Aulock 3301; SNG Slg. von Aulock 3305; SNG Kopenhagen XXVIII 735(1)

\section{Mysien:}

Attaia (98-117; 117-192; 193-211): SNG Slg. von Aulock 7203; SNG Slg. von Aulock 1070; Fitzwilliam 4139

Germe (100-200; 117-192;138-192; 238-244; Kaiserzeit): SNG Slg. von Aulock 7214; SNG Tübingen 2199; SNG Kopenhagen XXVII 124; SNG Kopenhagen XXVII 129; SNG Kopenhagen XXVII 122

Hadrianeia (117-138 bzw. 161; 193-211; 198-235): SNG Slg. von Aulock 1126; Fritze, Mysien 440; SNG Kopenhagen 19 166; Fritze, Mysien 441 Hadrianothera (117-138; 211-268): SNG Slg. von Aulock 1146; Fritze, Mysien 556 
Pergamon (-27-138; 40-6o; 100-200; 238-268; 258-26o): SNG Kopenhagen 19 446; RPC I 2374-2378; SNG Slg. Righetti (Bern) o748; BMC XII 137 Nr.235; SNG Kopenhagen 19460

\section{Phrygien:}

Aizanoi (-27-38; Chr. Geb.-100; 38 bzw. 41-54; 117-138; 200 bzw. 253-268; 250-276; Kaiserzeit): Imhoof, 190 Nr.7; SNG Slg. Righetti (Bern) 1125; RPC I 3097. 3104-3106; BMC XVII 24 Nr.6; SNG Kopenhagen XXIX 68; SNG Kopenhagen XXIX 66; SNG Slg. von Aulock 8302; SNG Kopenhagen XXIX 67

Akkilaion (238-244): Aulock, Phrygien I 1

Alia (98-117; 161-180): Aulock, Phrygien 1 39; Aulock, Phrygien 1 Nr.61

Ankyra (62/63; 69-96; 100-276; 138-161; 193-211 bzw.235): RPC I 3113A-B; SNG Slg. von Aulock 3425; SNG Slg. von Aulock 3426; SNG Slg. Lewis (Cambridge) 1515; BMC XVII 6o Nr.15; SNG Kopenhagen XXIX 130 Apameia (244-268; 251-253): Slg. Weber 7032; BMC XVII 91 Nr.132 Appia (98-117): SNG Slg. von Aulock 3519 Dionysopolis (221/222): Aulock, Phrygien II 88

Dokimeion (100-276; 193-211): SNG Kopenhagen XXIX 353; SNG München XXIV 177

Eumeneia (190 bzw. 193-230): Slg. Weber 7092; SNG Kopenhagen XXIX 389

Grimenothyrai (97 bzw. 117-138): Aulock, Phrygien II 329; Aulock, Phrygien II 395

Hadrianopolis (117-138): SNG Tübingen 3831

Hierapolis (98-117 bzw. 217; 118-222; 198-268; 218-222): SNG Kopenhagen XXX 413; SNG Kopenhagen XXX 414; SNG München XXIV 227; SNG Kopenhagen XXX 445; SNG Slg. von Aulock 3633

Hyrgaleis (198-235; 221/222; 240/241): Aulock, Phrygien I 333; Aulock, Phrygien I 350; Aulock, Phrygien 1357

Kadi (41-54; 117-138): SNG Kopenhagen XXIX 238; SNG Kopenhagen XXIX 239

Kibyra (81-96; 161-180; Kaiserzeit): SNG Slg. von Aulock 8395; SNG Tübingen 4095; SLG HUNTER S482,2

Kidyessos (178-187): SNG Slg. von Aulock 3758

Kolossai (253-268): Aulock, Phrygien II 543

Kotaion (68/69; 68-79; 253-268): RPC I 3223-3226; Slg. Weber 7071; I. Svoronos, Journal International d'Archéologie Numismatique 12(1909) 41 Nr. 1 
Laodikeia (138-161 bzw. 180; 198-217 bzw. 268): SNG Slg. von Aulock 3826; SNG München XXIV 361; Imhoof, GM S161,6; SNG Kopenhagen XXX 541 Okokleia (177-192; 238-244): Aulock, Phrygien 1 704; Aulock, Phrygien I 707

Peltai (138-192; 251-253; Kaiserzeit): BMC XVII 350 Nr.21; SNG Slg. Lewis (Cambridge) 1592; SLG WALCHER 2805

Prymnessos (79-81; 138-192; 193-238): Aulock, Phrygien II 855; Aulock, Phrygien 2 905; SNG Slg. von Aulock 3935

Sebaste (100-276; 198-268; Kaiserzeit): SNG Slg. von Aulock 3950; SNG Slg. von Aulock 3949; SNG Kopenhagen XXX 676

Synaos (69-192; 100-276; Kaiserzeit): SNG Kopenhagen XXX 700; SNG Slg. von Aulock 3963; Slg. Weber 7178

Synnada (Chr. Geb.-200; 138-161; 150-200): SNG Kopenhagen XXX 715; IMHOOF MG S413,159; SNG Slg. von Aulock 3976

Temenothyrai (161-180; 244-249; 253-268): SNG Slg. Righetti (Bern) 1229; BMC XVII 410 Nr.12; SNG Tübingen 4203

Tiberiopolis (98-117; 117-138): SNG München XXIV 497; SNG Slg. Lewis (Cambridge) 1575

Traianopolis (98-117; 117-138; 198-217): SNG Kopenhagen XXX 758; SNG Kopenhagen XXX 759; Aulock, Phrygien II 1424

Troas:

Abydos (-69): RPC I 2283

Ilion (68-69): RPC I 2317(1-6)

Pionia (117-161 bzw. 217): SNG Slg. von Aulock 7631; BMC XII 77 Nr.1

\section{Anhang II:}

Tabelle: Die Statuenprozession des Vibius Salutaris in Ephesos

\begin{tabular}{clll}
\hline Position & Darstellung & $\begin{array}{c}\text { Gewicht } \\
\text { (in g) }\end{array}$ & Material \\
\hline 1 & Traian & $?$ & Silber \\
2 & Plotina & 982 & Silber \\
3 & Artemis & 982 & Gold \\
4 & Synkletos & 1365 & Silber \\
5 & Boule & 1320 & Silber \\
6 & Artemis & 1965 & Silber \\
7 & Demos Rhomaion & $?$ & Silber \\
8 & Gerousia & $?$ & Silber \\
& & & (continued)
\end{tabular}


Tabelle (cont.)

\begin{tabular}{rlll}
\hline Position & \multicolumn{1}{c}{ Darstellung } & $\begin{array}{c}\text { Gewicht } \\
(\text { in g) }\end{array}$ & Material \\
& & $2101+\mathrm{x}$ & \\
\hline 9 & Artemis & 999 & Silber \\
10 & Hippikon Tagma & $?$ & Silber \\
11 & Ephebeia & Silber \\
12 & Artemis & $?$ & Silber \\
13 & Augustus & $?$ & Silber \\
14 & Phyle Sebaste & $?$ & Silber \\
15 & Artemis & $?$ & Silber \\
16 & Demos & $?$ & Silber \\
17 & Phyle Epheseon & $?$ & Silber \\
18 & Artemis & $?$ & Silber \\
19 & Androklos & $?$ & Silber \\
20 & Phyle Karenaion & $?$ & Silber \\
21 & Artemis & $?$ & Silber \\
22 & Lysimachos & $?$ & Silber \\
23 & Phyle Teion & $?$ & Silber \\
24 & Artemis & $?$ & Silber \\
25 & Euonymos & $?$ & Silber \\
26 & Phyle Euonymon & $996+\mathrm{x}$ & Silber \\
27 & Artemis & $?$ & Silber \\
28 & Pion & $?$ & Silber \\
29 & Phyle Bembeinaion & $?$ & Silber \\
30 & Athena Pammousos & 2315 & Silber \\
31 & Sebaste Homonoia Chrysophoros & 1965 & Silber \\
\hline
\end{tabular}

